\title{
The dynamic analysis of piping systems using pseudo-dynamic techniques
}

\author{
F J M Q de Melo*, J A O Carneiro, H R Lopes, J F Dias Rodrigues and J F Silva Gomes \\ New Materials and Experimental Methods, INEGI, Institute of Mechanical Engineering and Industrial Management, Porto, \\ Portugal
}

\begin{abstract}
This paper describes an application of pseudo-dynamic techniques to the dynamic analysis of piping structures. Essentially it consists in coupling a direct time integration algorithm, such as the Newmark method, to an experimental step. At each time step the integration algorithm generates a displacement vector of the structure, which is prescribed for the test specimen. This is mounted in a rigid test rig fitted with a set of displacement actuators and load cells at the level of the structure degrees of freedom. The load cells allow the reading of the internal restoring force vector, which is fed back to the direct time integration algorithm in an actual time step. Further calculations for the velocity and acceleration vectors will define a new structure configuration by evaluating a new displacement vector referred to the next time step. This procedure makes it possible to assess experimentally a realistic stress distribution at sections of complex shape piping parts. The method is a precise tool in dynamic analysis and, on being carried out in a quasi-static procedure, it operates with less expensive equipment than is necessary in real dynamic test.
\end{abstract}

Keywords: dynamic analysis, piping systems, pseudo-dynamic techniques

\section{INTRODUCTION}

The broad application areas for piping systems include in the project the influence of time-dependent load actions, following specifications in design codes. This is the case of seismic forces or those resulting from the influence of reciprocating machines included in the system. The case of seismic forces has a leading importance in this type of structure, as catastrophic effects may arise in the system, given the high amplitude of the displacements. Damage mechanisms occur in piping systems frequently at the level of supports, releasing the structure from necessary restrictions and contributing to high-level stress fields at piping details particularly vulnerable to overloads. This is the case of pipe elbows or tee connections, where these accessories undergo a considerable section distortion due to ovalization under in-plane or out-of-plane bending effects.

Relevant work in the design of seismic proof constructions has involved research techniques involving pseudodynamic methods. Aktan [1], Aktan and Hashish [2], Beck and Jayakumar [3] and Akao and Watabe [4] have devel-

The MS was received on 21 February 2000 and was accepted after revision for publication on 19 April 2001.

* Corresponding author: Departamento de Engenharia Mecânica e Gestão Industrial, Faculdade Engenharia, Universidade do Porto, Rua Dr Roberto Frias S/N, 4200-465 Porto, Portugal. oped seismic resistant structures after pseudo-dynamic tests performed on reduced-scale models. These researchers have also studied the influence of several damping models on the reduction in the vibration amplitude, having developed dissipation energy motion supports. In the research area of buckling mechanisms of axially loaded columns submitted to transverse seismic actions, contributions have been made by Miki and co-workers [5-17] at the Daido Institute of Technology, Nagoya, Japan. For this purpose, pseudo-dynamic techniques were selected to define the damage model of axially loaded columns and submitted to time-variable transverse loads.

Significant contributions to the application of these techniques to the study of seismic actions on structures have been made by the European Laboratory for Structural Assessment (ELSA), part of the Joint Research Centre (JRC) located at Ispra (VA), Italy. In these research facilities, pseudo-dynamic tests were carried out with fullor reduced-scale models assembled in a huge reaction wall test rig. The simulation of seismic actions has been performed at ELSA, JRC. In these research facilities the pseudo-dynamic tests were carried out with full- or reduced-scale models, assembled in a huge reaction wall test rig. At the JRC, Pinto and co-workers [18-23] have presented contributions on the stress analysis and damage mechanisms in civil constructions.

The application of pseudo-dynamic techniques is still expanding, meeting increasing interests in the scope of 